\title{
Estimation of reproduction numbers of COVID-19 in typical countries and epidemic trends under different prevention and control scenarios
}

\author{
Chen $\mathrm{Xu}^{1, *}$, Yinqiao Dong ${ }^{2, *}$, Xiaoyue $\mathrm{Yu}^{1, *}$, Huwen Wang ${ }^{1, *}$, Lhakpa Tsamlag ${ }^{1, *}$, Shuxian Zhang ${ }^{1, *}$, Ruijie Chang ${ }^{1, *}$, \\ Zezhou Wang ${ }^{3, *}$, Yuelin $\mathrm{Yu}^{1, *}$, Rusi Long ${ }^{1, *}$, Ying Wang ${ }^{1}$, Gang Xu ${ }^{1}$, Tian Shen ${ }^{1}$, Suping Wang ${ }^{1}$, Xinxin Zhang $\left.(\bowtie)\right)^{4}$, \\ Hui Wang $(\bowtie)^{1}$, Yong Cai $(\bowtie)^{1}$ \\ ${ }^{1}$ School of Public Health, Shanghai Jiao Tong University School of Medicine, Shanghai 200025, China; ${ }^{2}$ Department of Environmental and \\ Occupational Health, School of Public Health, China Medical University, Shenyang 110122, China; ${ }^{3}$ Department of Cancer Prevention, \\ Shanghai Cancer Center, Fudan University; Department of Oncology, Shanghai Medical College, Fudan University, Shanghai 200025, \\ China; ${ }^{4}$ Research Laboratory of Clinical Virology, National Research Center for Translational Medicine (Shanghai), Ruijin Hospital and \\ Ruijin Hospital North Affiliated to Shanghai Jiao Tong University School of Medicine, Shanghai 200025, China
}

(C) Higher Education Press 2020

\begin{abstract}
The coronavirus disease 2019 (COVID-19) has become a life-threatening pandemic. The epidemic trends in different countries vary considerably due to different policy-making and resources mobilization. We calculated basic reproduction number $\left(R_{0}\right)$ and the time-varying estimate of the effective reproductive number $\left(R_{t}\right)$ of COVID-19 by using the maximum likelihood method and the sequential Bayesian method, respectively. European and North American countries possessed higher $R_{0}$ and unsteady $R_{t}$ fluctuations, whereas some heavily affected Asian countries showed relatively low $R_{0}$ and declining $R_{t}$ now. The numbers of patients in Africa and Latin America are still low, but the potential risk of huge outbreaks cannot be ignored. Three scenarios were then simulated, generating distinct outcomes by using SEIR (susceptible, exposed, infectious, and removed) model. First, evidence-based prompt responses yield lower transmission rate followed by decreasing $R_{t}$. Second, implementation of effective control policies at a relatively late stage, in spite of huge casualties at early phase, can still achieve containment and mitigation. Third, wisely taking advantage of the time-window for developing countries in Africa and Latin America to adopt adequate measures can save more people's life. Our mathematical modeling provides evidence for international communities to develop sound design of containment and mitigation policies for COVID-19.
\end{abstract}

Keywords reproduction number; SEIR model; COVID-19; estimate

\section{Introduction}

The outbreak of the coronavirus disease 2019 (COVID-19) has affected more than two hundred countries, areas or territories worldwide [1]. World Health Organization (WHO) announced COVID-19 is a pandemic [2]. As of March 28, 2020, 570947 confirmed cases of the COVID-

Received April 1, 2020; accepted April 5, 2020

Correspondence: Yong Cai, caiyong202028@hotmail.com; Hui Wang, huiwang@shsmu.edu.cn; Xinxin Zhang, zhangx@shsmu.edu.cn

${ }^{*}$ These authors contributed equally to this manuscript.
19 have been reported and 26486 people have lost their lives worldwide according to the data from WHO [1]. Europe and United States of America have now become the new epicenters of the pandemic, with more reported cases and deaths than the rest of the world combined, apart from China [1,3]. The situations in the Africa and Latin America are also not optimistic. Urgent and effective joint actions against COVID-19 should be considered as the top priority of the international communities, as reflected by the voice of the G20 conference held on March 26, 2020. Comprehensive approaches were implemented in some neighbor countries of China such as the Republic of Korea, Singapore, and Japan [4-6]. The epidemiological and clinical studies have proven that early detection, isolation, 
tracing close contact, and treating suspected/mild cases are effective in slowing down transmission, flattening the epidemic curve, and reducing the mortality and morbidity rates among severe cases [2]. Experiences from China and these countries provided valuable reference for other countries to prevent and control COVID-19 [7]. Evidence has been accumulated that this apparently very devastating pandemic can be contained and the chains of transmission can be blocked if sufficient efforts and measures of prevention and control are timely undertaken in spite of unavoidable temporary economic cost.

The infectious disease dynamics model (Susceptible, Exposed, Infectious, and Removed model (SEIR model)) is widely used to model and predict the number of infections of infectious diseases such as SARS and COVID-19 epidemic [8-12]. Two recent studies used SEIR model to forecast the phase- and region-adjusted epidemic trend of COVID-19 in Wuhan, Hubei Province and regions outside Hubei Province in China and the results correlated well with actual data owing to an evidence-based gradual tightening of public health intervention $[13,14]$. Over the past week, only three local cases were reported from the community infection in Hubei, Henan, and Zhejiang provinces, whereas early detection of imported cases has become a challenge for China in consolidating the results of containment [15]. The values of variables including basic reproduction number $\left(\mathrm{R}_{0}\right)$, effective reproduction number $\left(\mathrm{R}_{\mathrm{e}}\right)$, and time-varying estimate of $R_{e}\left(R_{t}\right)$ are vital for the model. $R_{0}$ plays a crucial role in predicting the prevalence of the infectious disease which represents the average number of secondary cases generated by a primary infector [16]. The values of $\mathrm{R}_{0}$ in China were reported 2.2, 2.6, 3.8, or even 6.47 according to different researches [11,16-18]. The epidemic trends in different regions around the world are significantly different due to the great difference in the design and implementation of prevention and control measures [19]. Estimates of both $\mathrm{R}_{0}$ and $\mathrm{R}_{\mathrm{t}}$ in different scenarios will enable a better forecast of global epidemic trends.

In what follows we took a maximum likelihood method and a sequential Bayesian approach to estimate the values of $R_{0}$ and $R_{t}$ of several countries affected by COVID-19, we also used the SEIR model with $R_{e}$ in different stages to simulate and compare the epidemic trends under three scenarios with different levels of public health intervention measures. Furthermore, we proposed some theoretical references and possible choices of feasible prevention and control approaches for the decision-making in a countryor region-specific context.

\section{Materials and methods}

The COVID-19 epidemic data were collected from WHO and other related official websites. We selected large population countries with a relatively high prevalence of COVID-19 for data analysis. Therefore, 12 countries with more than 40 million people each were selected from different continents, including Republic of Korea, Japan, Italy, Spain, France, Germany, the UK, United States of America, Islamic Republic of Iran, Algeria, South Africa, and Argentina [20]. Data collected about different countries were from the date when the first case in the country was reported to March 23, 2020. We employed prior estimated distribution with a mean of 7.5 and a standard deviation of 3.4 for the serial interval defined as the time between infections in consecutive generation [17]. Given daily confirmed cases from 12 countries over consecutive time units (days) and serial interval distribution, the basic reproduction number $\left(\mathrm{R}_{0}\right)$ was estimated by using maximum likelihood method [21-23]. MetropolisHastings Monte Carlo method was used to estimate the values $95 \%$ confidence interval $(\mathrm{CI})$ of basic reproduction number $\left(\mathrm{R}_{0}\right)$. We also estimated the time-varying estimate of the effective reproduction number $\left(\mathrm{R}_{\mathrm{t}}\right)$, which means average number of secondary cases that primary case could generate at time $t$ when conditions reminded [24], using the sequential Bayesian method to measure the potential transmission capacity of SARS-CoV-2 and intervention effect. In sequential Bayesian framework, the package in $\mathrm{R}$ software calculated the time-varying estimate of the effective reproduction number $\left(\mathrm{R}_{\mathrm{t}}\right)$ automatically using the prior distribution on each new day which is posterior distribution from the previous day. The sequential Bayesian method is adopted widely recently because it can estimate the real-time trends of effective reproduction number with joint effects of random process of personnel mobility and imported cases taken into consideration [25]. These estimated methods mentioned above were based on the collected data. Sensitivity analysis was conducted by varying the parameters of serial interval distribution.

We simulated and predicted the dynamic epidemic trends using the phase-adjusted SEIR model in three scenarios assumed based on actual epidemic status and the prevention measures and policies adopted by different countries [14]. In the first scenario, prevention and control measures were implemented in a prompt and effective way. It has relatively high level of access to the operation readiness capacities in country level and high level of adherence to public health interventions among the demos [26]. In the second scenario, at the early stage, prevention and control measures were loose at social level and awareness of self-prevention was weak at individuals' level. As the epidemic scale of COVID-19 progressed, prevention and control measures were strengthened. The third scenario represents the situation in countries which were still at the beginning of the epidemic but at huge risk of large scale of outbreak. Poor social-economic situations and low operational readiness capacities in these countries might prevent necessary public health policies from being 
implemented. Insufficient healthcare resources may rapidly collapse like the situation during Ebola outbreak in 2014 [26]. In the SEIR model, the median incubation period was assumed 7.5 days and the infectious period was assumed 12 days [14,21,27]. The set of initial values of Susceptible, Exposed, Infectious and Removed cases were based on the population and confirmed cases data collected from the World Bank and WHO database $[20,28]$. In the first and second scenarios, we forecasted the trends of COVID-19 based on the estimated $R_{e}$. The change of $R_{e}$ is estimated on average according to $\mathrm{R}_{t}$ change in the time period that has occurred, and the change of $R_{e}$ in the future is estimated according to the multi-stage change [14]. In the third scenario, $R_{e}$ was assumed equal to $R_{0}$ to show the possible epidemic trend without any effective prevention and control measures. This model was constructed under some assumptions mentioned in the limitation section.

Estimation of $R_{0}$ and $R_{t}$ and corresponding sensitivity analysis, and construction of SEIR model were conducted using the $\mathrm{R}_{0}$ package and deSolve package, respectively in $\mathrm{R}$ software (version 3.6.3).

\section{Results}

\section{Estimation of basic reproduction numbers of COVID-19 epidemic in 12 typical countries}

The value of $\mathrm{R}_{0}$ in Republic of Korea was the lowest $(1.593,95 \%$ CI $1.582-1.604)$, while the highest in United States of America (8.213, 95\% CI 8.139-8.288) (Fig. 1).

The sensitivity analysis with various serial interval distributions was conducted, which showed the trends were robust among the different countries (the detailed information is supplied in supplementary material).

Time-varying estimations of the effective reproduction number of COVID-19 epidemic in 12 typical countries

In Italy, a rapid increase of $R_{t}$ after February 20, 2020 was detected and followed by a fast decrease in a few days. In Spain, the $\mathrm{R}_{\mathrm{t}}$ grew beyond 2 on February 26, 2020 and fluctuated slightly ever since. In United States of America, $R_{t}$ values climbed slowly since February 20, 2020. In South Africa, $R_{t}$ values fluctuated around 4 since the first reported case. More details and other countries' timevarying estimations of $R_{t}$ were showed in Figs. 2 and 3.

\section{Estimated epidemic trends of COVID-19 under different scenarios of preventive and control measures}

\section{Scenario 1}

In this scenario, where prevention and control measures

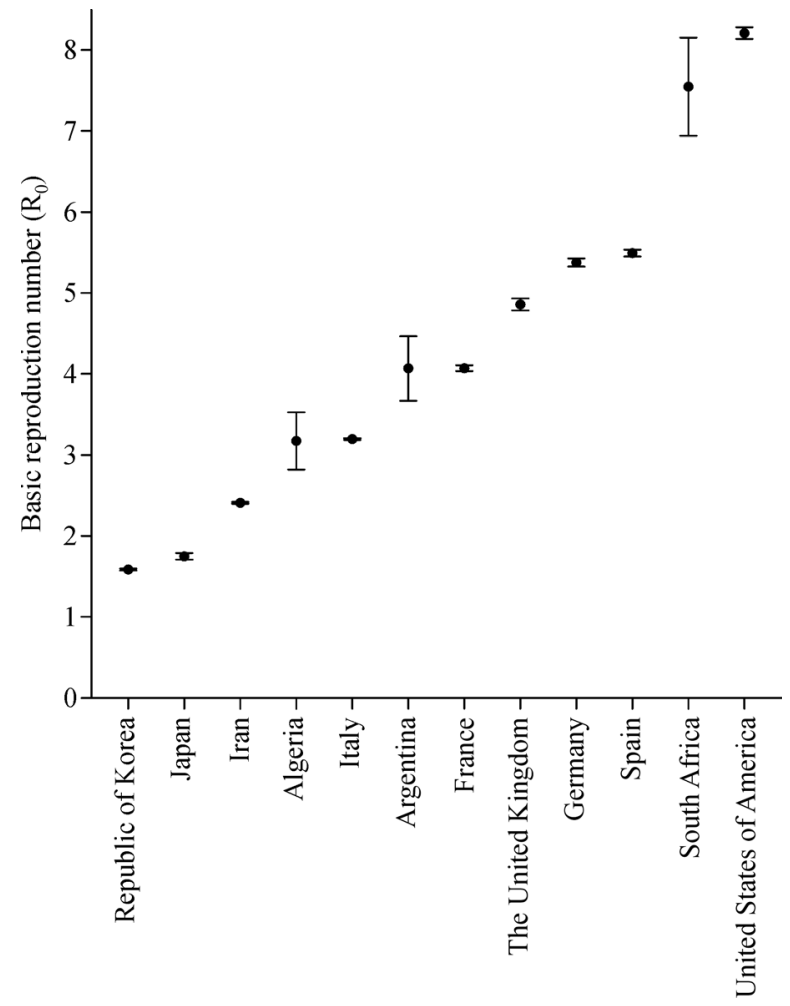

Fig. 1 Estimation of basic reproduction numbers of COVID-19 epidemic in 12 typical countries. The maximum likelihood method was used to estimate the values of basic reproduction number $\left(\mathrm{R}_{0}\right)$ of COVID-19 in 12 mainly affected countries from different continents. The dots and vertical bars represent the value of $\mathrm{R}_{0}$ and $95 \%$ confidence interval, respectively.

were implemented in prompt and effective way, the epidemic trend was calculated based on a country with 120 million susceptible people (Fig. 4). The peak of the number of infections would possibly occur in mid-to-late April 2020. The highest number of infections would be 2412 on April 20, 2020 in our simulated model, accounting for $1.91 / 100000$ of the whole population.

\section{Scenario 2}

In the second scenario, the prevention and control measures were loose at the early stage with a temporary rising $R_{e}$ but were significantly strengthened as the epidemic was tremendously progressing (Fig. 5). Our estimation was performed by employing a country with a population of 60 million where the epidemic started in early January 2020. The peak of the number of infected people would occur in late April 2020 if effective measures could be vigorously implemented since mid-March 2020 . The number of confirmed patients would be high but a turning point could eventually appear with 209517 estimated cases on April 27, 2020 in our simulated model, accounting for $0.35 \%$ of the whole population. 

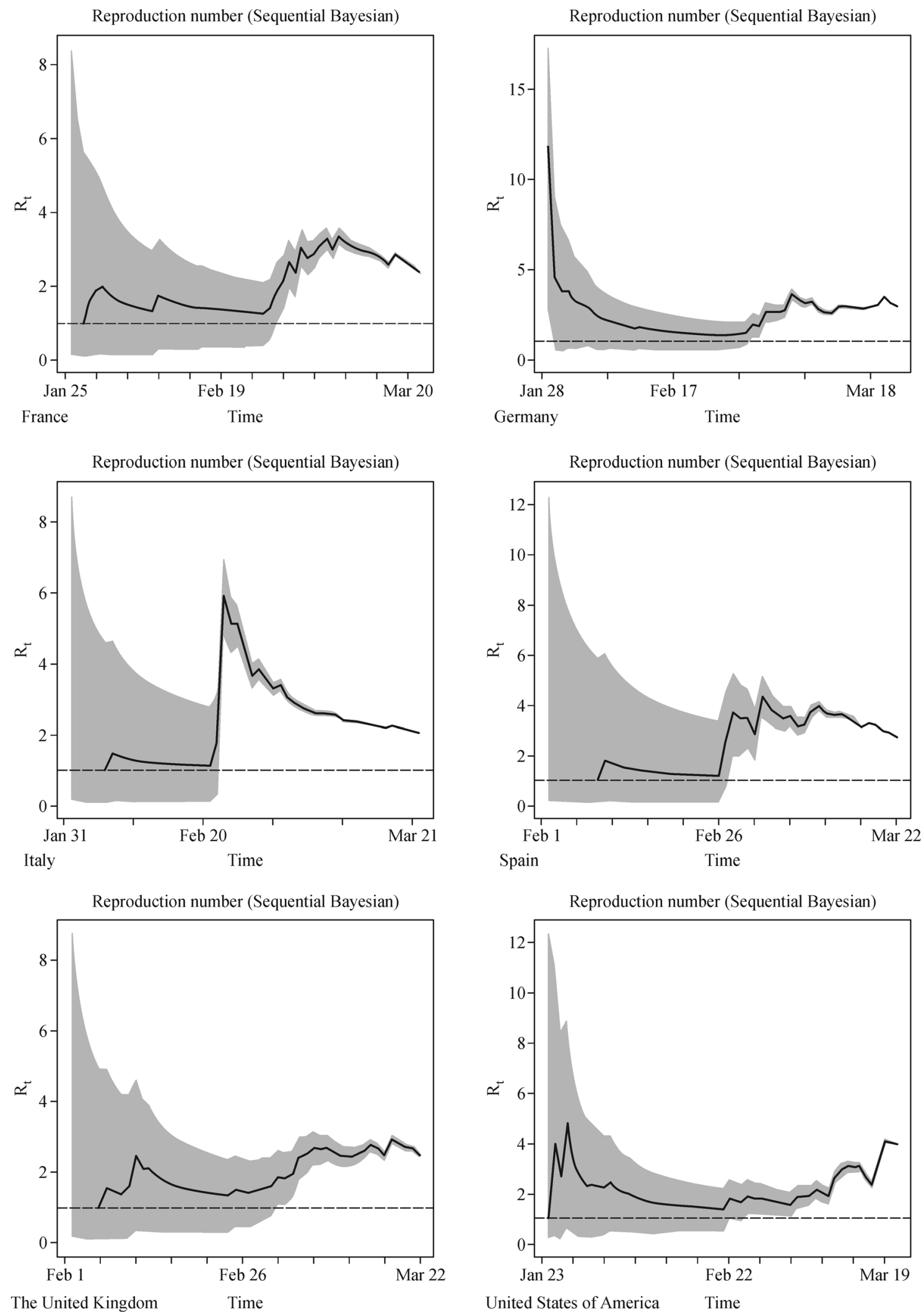

Fig. 2 Time-varying estimations of the effective reproduction number in selected European and North American countries. The sequential Bayesian method was used to calculate time-varying estimations of the effective reproduction number $\left(R_{t}\right)$ in Italy, France, Spain, Germany, the UK, and United States of America. Light gray ribbon means 95\% confidence interval. The dotted line indicates the target value of 1 for the effective reproduction number required for control. 

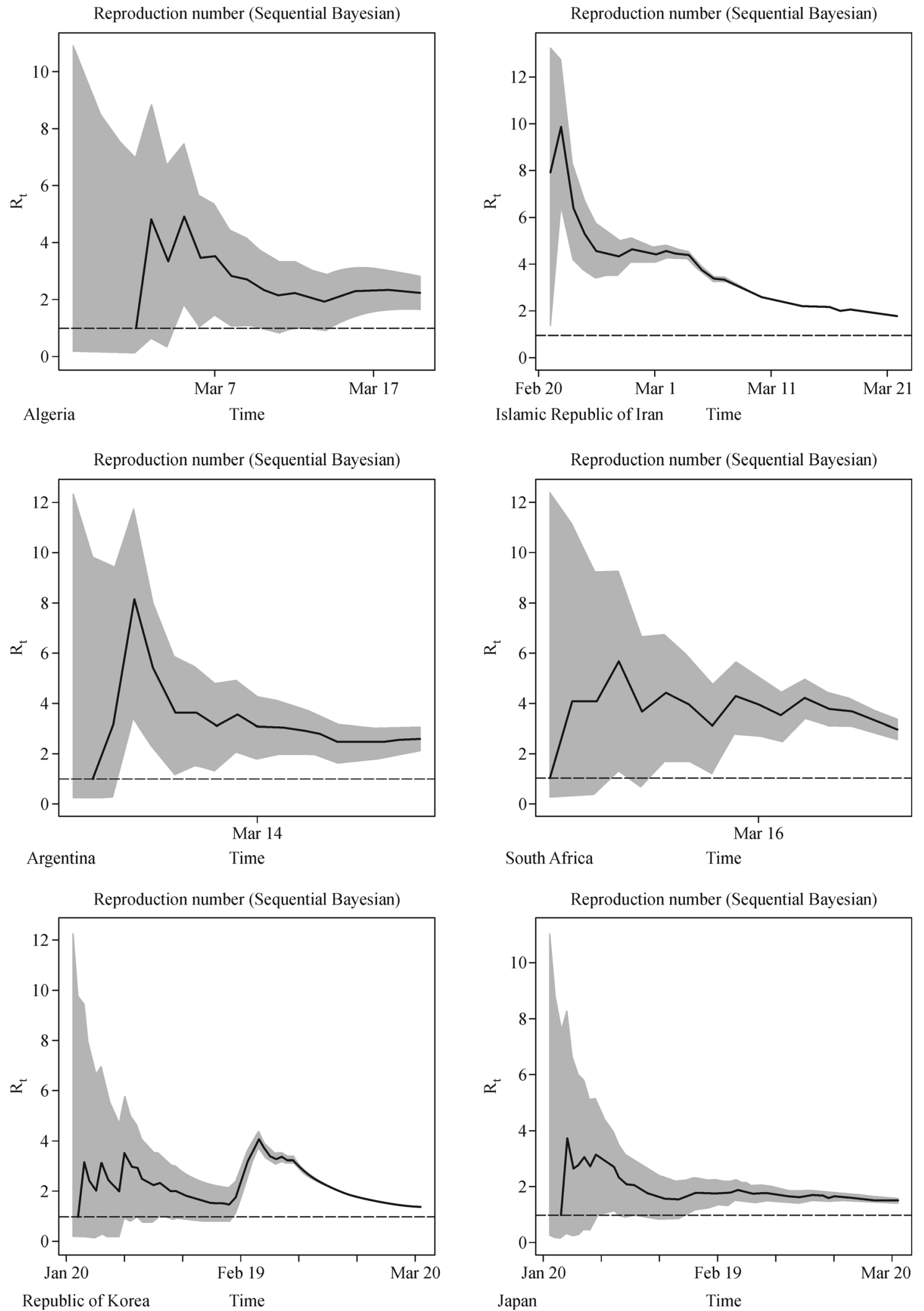

Fig. 3 Time-varying estimations of the effective reproduction number in selected Asian, African, and Latin American countries. The sequential Bayesian method was used to calculate time-varying estimations of the effective reproduction number $\left(\mathrm{R}_{\mathrm{t}}\right)$ in Japan, Republic of Korea, Islamic Republic of Iran, South Africa, Algeria, and Argentina. Light gray ribbon means 95\% confidence interval. The dotted line indicates the target value of 1 for the effective reproduction number required for control. 


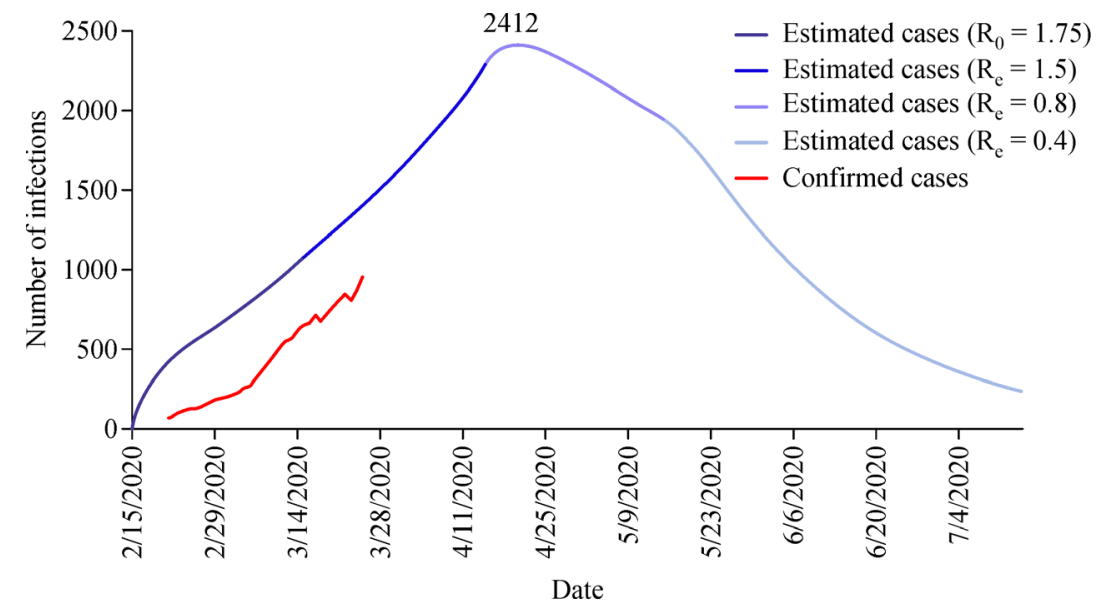

Fig. 4 Estimated epidemic trend of COVID-19 under scenario 1. Different colors were used to distinguish the trends of estimated and confirmed cases as well as different stages of estimated tendency divided by the values of basic reproduction number $\left(\mathrm{R}_{0}\right)$ and effective reproduction number $\left(\mathrm{R}_{\mathrm{e}}\right)$. The highest number of infections would be 2412 on April 20, 2020 in our simulated model.

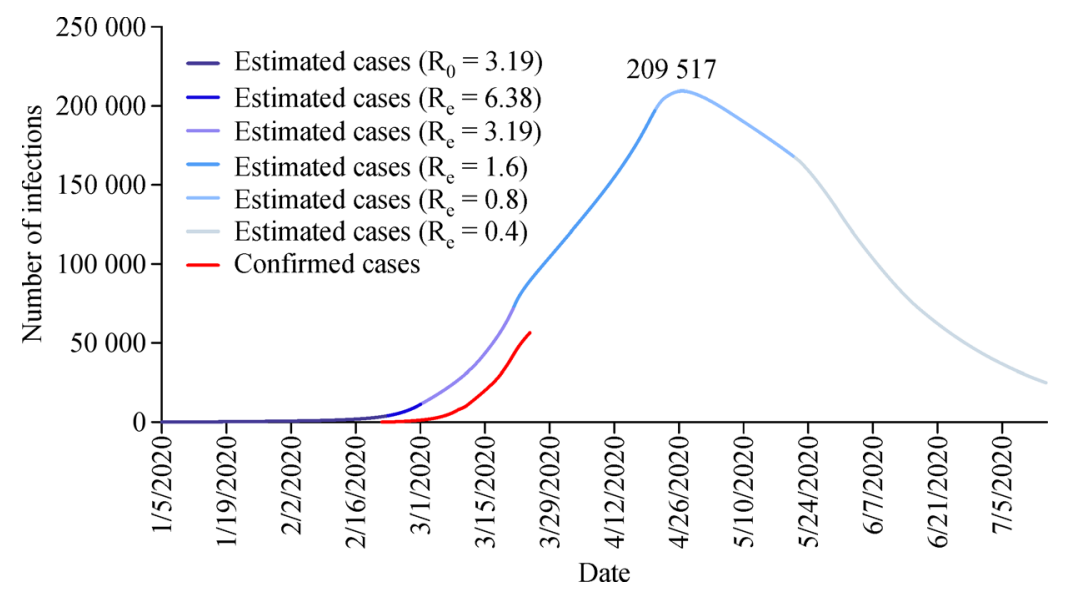

Fig. 5 Estimated epidemic trend of COVID-19 under scenario 2. Different colors were used to distinguish the trends of estimated and confirmed cases as well as different stages of estimated tendency divided by the values of basic reproduction number $\left(\mathrm{R}_{0}\right)$ and effective reproduction number $\left(\mathrm{R}_{\mathrm{e}}\right)$. The highest number of infections would be 209517 on April 27, 2020 in our simulated model.

\section{Scenario 3}

The third scenario mainly simulated the epidemic trends in part of African and Latin American regions. We took two relatively large countries in North Africa (country A1) and South America (country A2), respectively having a population of 42 million and 44 million as examples. Currently, the epidemic is at a quite early stage in both countries. As of March 28, 2020, only 367 confirmed cases were reported in Country A1 while the number of confirmed cases was 589 in Country A2. Nevertheless, the number of infections would keep rising as time goes by if no strict public health intervention was implemented and the peak would not occur before July 2020 in both countries. Using our model, the number of infected people could reach 16307 in the former and 71770 in the latter, respectively by May 31, 2020 (Fig. 6). On the other hand, if time opportunities could be grasped, like in provinces in China outside the epicenter City of Wuhan and Hubei Province, through determined public health intervention, the curves of epidemic could be reshaped with decreasing $R_{t}$.

\section{Discussion}

During the period of a global pandemic, when reported cases are rising at a high speed, forecasting tools are of crucial importance for public health policy-making $[29,30]$. The values of $R_{0}$ of nations we estimated in European and North American regions are all above 3, 


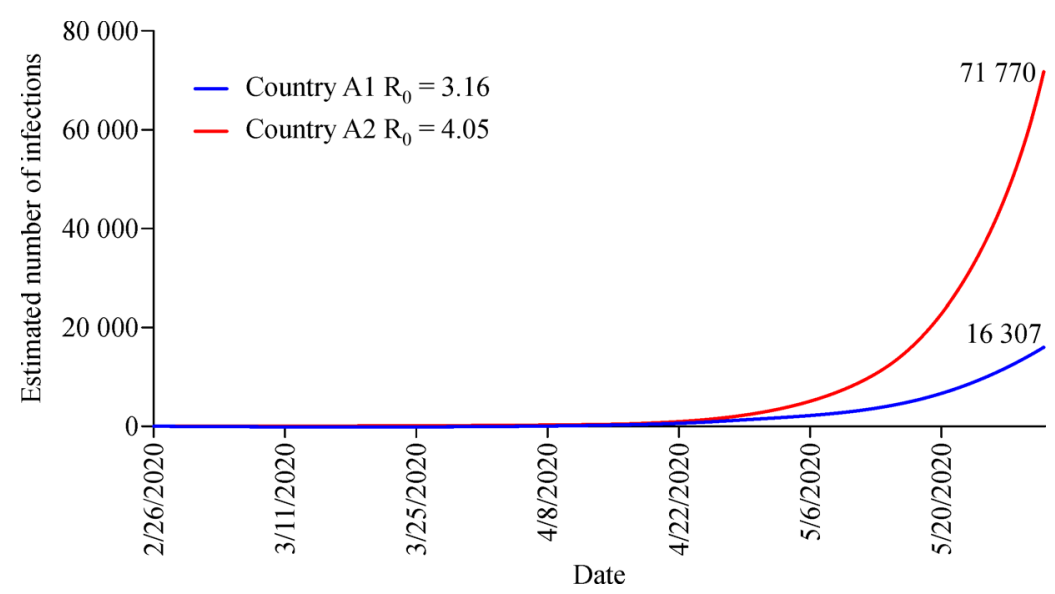

Fig. 6 Estimated epidemic trend of COVID-19 under scenario 3. The estimated number of infections will keep rising as time goes by with unchangeable basic reproduction number $\left(\mathrm{R}_{0}\right)$ in both country $\mathrm{A} 1$ and country $\mathrm{A} 2$. The number of infections would reach 16307 and 71770 in country A1 and country A2, respectively by May 31, 2020.

suggesting the vulnerability of these countries to the disease and the serious epidemic situations. The outcomes of the subsequent epidemics still depend on the intensity of prevention and interventions as well as the public awareness. $\mathrm{R}_{0}$ values are below 2 in Japan and Republic of Korea, representing a relatively lower transmissibility thanks to efficient containment measures along with persistently declining $\mathrm{R}_{\mathrm{t}}$. It is well accepted that for public health measures to take effects, early phase interventions are of key importance. Therefore, though the total confirmed cases in Africa and Latin America are relatively low, probably because the epidemic is still in early stage, the situations might not be optimistic if no caution was taken. As expected from our estimations, due to high $\mathrm{R}_{0}$ values and unsteady fluctuation of $R_{t}$, COVID-19 would quickly spread out in these regions. Of note, weak healthcare systems, lack of medical resources, poor public health conditions, and untimely detection are all risk factors that could lead to worse outcome in low-income countries if no appropriate strategies were adopted [26].

We predicted three possible trends for global epidemic based on the estimated $\mathrm{R}_{0}$ and $\mathrm{R}_{\mathrm{e}}$ as well as current epidemic situations in different countries. Scenario 1 is undoubtedly the best option: rapid response of containment is implemented once a nation starts to be affected by COVID-19. In the second scenario, weak awareness of the public and insufficient control measures at the beginning of the epidemic resulted in rapid transmission speed and a heavy casualty. Infection of a large number of healthcare workers would make the hospitals sources of human-tohuman transmission and could cause the panic of the society. However, the adjustment of governmental response to the outbreak, with the support of international society, could reshape the epidemic profile and the negative effect of COVID-19 could be gradually mitigated. Currently, a major concern of the international society is about the scenario 3, in which under-developed countries, some of them still facing protracted armed conflict or with very vulnerable social-economic conditions, could lose the narrowing time window for the development and implementation of sound public health measures. Weak healthcare system could risk to collapse once an unmet need of service was emerging. Instead, wise actions taking advantage of the current time window, though narrowing down with each passing day and week, might provide efficient protection of people's life.

What can we do quickly to improve the ability of containment and mitigation of countries in the scenario 2 and prevent a large number of under-developed countries suffering the poor outcome in scenario 3 ? We would like to propose some basic public health policies, which should be adapted to the context of distinct cultures and socioeconomic conditions of different countries.

First, the concept that COVID-19 is a containable disease should be strengthened. Experience of China and some East Asian countries have provided strong evidence for this concept $[4,5,31]$, although the serological investigation is needed to rule out the possibility for the epidemic to be transformed into seasonal community pneumonia [32]. The determination of decision-makers and public awareness constitute the foundation of a quick, efficient, and people-centered policy design and implementation so that a decline of the values of $R_{t}$ is possible. For the moment, in countries still facing real epidemic challenge, traditional control measures such as the mass facemask wearing and correct hand washing, rest at home for at least 3 to 4 latency period of time (around one month), social distancing via suspension of public gathering activities and close of school, even the lockdown of transport from and to the epicenter cities or regions as well as the suspension of public and private traffics, have been shown to be successful in preventing human-to- 
human transmission [7]. Given the initial neglect of the epidemic outbreak, restoration and expanding of industries for protective materials and test reagents, large-scale screening and subsequent isolation/appropriate treatment of confirmed patients, as tracing close contacts at community level, and preventing hospital outbreaks should be applicable to all nations [33].

Second, tiered medical services for mild (or nonsymptomatic), moderate, severe, and critical cases should become a good strategy to make a full play of the roles of the primary healthcare, secondary hospital (or some shelter hospitals in open spaces such stadiums, museums, big conference halls of universities/schools) and tertiary hospital, especially those specialized for the treatment of COVID-19. For severe or critical patients, the principles of "Four Togethers" practiced in China could be taken as references: i.e., to hospitalize patients together in a limited number of hospitals with sufficient intensive care (ICU) units, deploy medical experts together in these centers, put resources together including equipment and effective drugs, and get patients treated together according to standardized protocols for COVID-19 [7]. Distinct treatment strategies should be used in different countries: countries with good primary healthcare system can derive greater benefits from community doctors, who are able to share a large amount of the burden on hospitals by taking care of patients with mild or moderate clinical symptoms. On the other hand, shelter hospitals can be temporarily established in cities or countries lacking enough welltrained family doctors to provide care to mild and moderate cases. As a result, the tertiary hospitals can be devoted to the treatment of severe cases. In addition to the supportive care using equipment such as ventilators, extracorporeal membrane oxygenation (ECMO), and continuous renal replacement therapy [34], specific life-saving treatment protocols have been developed, such as the combined use of convalescent plasma from recently recovered patients of COVID-19 and tocilizumab, a monoclonal antibody antagonist of interleukin 6 receptor (IL-6R). These two agents can target the SARS-CoV-2 and the aberrant cytokine release syndrome, respectively [35].

Third, clinical trials of vaccines for SARS-CoV-2 should be encouraged [36]. Research and development of vaccines against the viral pathogen will be of great value for protecting susceptible population in all countries, especially the elderly with non-communicable chronic diseases. Vaccines will be particularly useful to help countries with shortage of medical resources so that devastating situations can be avoided. Meanwhile, a large number of clinical trials of small molecule anti-viral drugs or drugs with potential effect in regulating immune system are ongoing [37]. Stem cell therapy is also carried out [38]. Coordinated trials are required to avoid abuse of precious clinical resources, whereas knowledge- and mechanismbased drug trials should be advocated.
Fourth, the education on general knowledge and skills of the epidemic control and prevention should be integrated into distinct country context. The speed of viral spreading is influenced by the local custom, such as the attitude toward facemask wearing. In some countries, governments are working with leaders of religious institutions or with non-government organizations (NGO) to facilitate the dissemination of the knowledge on the containment of COVID-19 into each family $[39,40]$. The staff and volunteers of humanitarian organizations such as Red Cross and Red Crescent societies should be mobilized to take care of the most vulnerable at the grassroots level. The use of military or police forces to ensure the social safety and supply of basic needs such as food, clean drinking water and healthcare service to the people is a choice in some countries.

Fifth, solidarity should be emphasized well beyond the different political opinions among different countries or different social groups within a country [31]. Viral transmission respects no borders or ethnic groups and COVID-19 is a common enemy of the mankind. The principles of transparency of the information on epidemic trends, the sharing of knowledge about the pathogenesis, and the exchanges of the best practice in terms of effective containment and treatment should be followed. On the other hand, stigmatization and prejudice must be opposed according to the International Health Regulation by World Health Organization [41]. International cooperation in the provision of protective materials and in the research and development of effective vaccines and drugs should be further encouraged.

Finally, we acknowledged some limitations of this study. First, the estimation of $\mathrm{R}_{0}$ depends on the serial interval. Here we assumed 7.5 days for all 12 countries due to limited information about the disease in different nations [17]. However, partly due to difference in control strategies among different nations, serial intervals are diverse in effect. The stability and accuracy of $R_{0}$ may be biased. Next, the SEIR model was based on a few essential assumptions (e.g., no multiple zoonotic sources of the virus, no transmission ability during incubation period, etc.), which may also be the inherent limitations of the model [42]. Usually, the SEIR model is used to estimate the epidemic in a closed crowd, while different degrees of domestic or international population migrations may lead to discrepancies between the estimates of this model and the actual situations. The accuracy of the estimation model depends largely on the accuracy of the parameters used (S, E, I, R, $\beta, \sigma$, and $\gamma$ ). Furthermore, hypothesis was raised by some researchers that the trend of COVID-19 could be influenced by temperature and relative humidity. The fact that relatively lower numbers of cases in African and South American countries could be in part ascribed to the warm and humid conditions. If this is true, alarming should be made for possible worsening of epidemic in Southern 
Hemisphere countries with change of seasons [43]. Finally, the data used to calculate $R_{0}$ and $R_{t}$ values of 12 countries and simulate SEIR model in three different scenarios were only from WHO's daily situation reports of COVID-19 without any epidemiological investigations. The accuracy and timeliness of the data online maybe affected by patients' diagnosis time and the scale of nucleic acid testing in different countries to some extent, which in turn influences the accuracy of the results. Despite the limitations mentioned above, the estimations we made in the present work including the $\mathrm{R}_{0}$ and $\mathrm{R}_{\mathrm{t}}$ of several typically affected nations and the outbreak size of COVID19 in different scenarios are not accurate predictions but may offer references for worldwide epidemic status analysis. These estimates may also allow a smarter design of public health policies in a country- and/or culturecontext adapted manner.

\section{Acknowledgements}

This work is funded by Medicine and Engineering Interdisciplinary Research Fund of Shanghai Jiao Tong University (No. YG2020YQ06), the National Key Research and Development Project (Nos. 2018YFC1705100, 2018YFC1705103, and 2018YFC2000700) and the National Natural Science Foundation of China (Nos. 71673187 and 81630086), the Key Research Program (No. ZDRW-ZS-2017-1) of the Chinese Academy of Sciences, Innovative research team of high-level local universities in Shanghai. We acknowledge all healthcare workers involved in the diagnosis, treatment, and prevention of COVID-19 all around world. We thank WHO and other institutes for coordinating data collection for patients with COVID-19.

\section{Compliance with ethics guideline}

Chen Xu, Yinqiao Dong, Xiaoyue Yu, Huwen Wang, Lhakpa Tsamlag, Shuxian Zhang, Ruijie Chang, Zezhou Wang, Yuelin Yu, Rusi Long, Ying Wang, Gang Xu, Tian Shen, Suping Wang, Xinxin Zhang, Hui Wang, and Yong Cai declare no competing interests. The data sets analyzed for this study can be found in the reports of the World Health Organization (WHO) (https://www.who.int/emergencies/diseases/novel-coronavirus-2019/situation-reports/). This manuscript does not involve a research protocol requiring approval by the relevant institutional review board or ethics committee.

Electronic Supplementary Material Supplementary material is available in the online version of this article at https://doi.org/ $10.1007 / \mathrm{s} 11684-020-0787-4$ and is accessible for authorized users.

\section{References}

1. WHO. Coronavirus disease 2019 (COVID-19) Situation Report - 68. 2020. https://www.who.int/docs/default-source/coro- naviruse/situation-reports/20200328-sitrep-68-covid-19.pdf?sfvrsn $=384$ bc74c $\_8$ (accessed March 28, 2020)

2. WHO. WHO Director-General's opening remarks at the media briefing on COVID-19-11 March 2020. 2020. https://www.who. $\mathrm{int} / \mathrm{dg} /$ speeches/detail/who-director-general-s-opening-remarks-atthe-media-briefing-on-covid-19---11-march-2020 (accessed March 27, 2020)

3. WHO. Coronavirus disease 2019 (COVID-19) Situation Report-54. 2020. https://www.who.int/docs/default-source/coronaviruse/situation-reports/20200314-sitrep-54-covid-19.pdf?sfvrsn $=$ dcd46351_8 (accessed March 27, 2020)

4. Choi JY. COVID-19 in South Korea. Postgrad Med J 2020. [Epub ahead of print] doi: 10.1136/postgradmedj-2020-137738

5. Ministry of Health, Singapore. Tighter measures to minimise further spread of COVID-19. 2020. https://www.moh.gov.sg/news-highlights/details/tighter-measures-to-minimise-further-spread-of-covid19 (accessed March 27, 2020)

6. Li LF. Novel coronavirus pneumonia: Japan's internal worries and countermeasures. 2020. https://3g.163.com/news/article_cambrian/ F7VCRUK10519D88G.html (in Chinese) (accessed March 27, 2020)

7. Zhang S, Wang Z, Chang R, Wang H, Xu C, Yu X, Tsamlag L, Dong Y, Wang H, Cai Y. COVID-19 containment: China provides important lessons for global response. Front Med 2020; 14(2): 215219

8. Cori A, Boëlle PY, Thomas G, Leung GM, Valleron AJ. Temporal variability and social heterogeneity in disease transmission: the case of SARS in Hong Kong. PLOS Comput Biol 2009; 5(8): e1000471

9. Fukutome A, Watashi K, Kawakami N, Ishikawa H. Mathematical modeling of severe acute respiratory syndrome nosocomial transmission in Japan: the dynamics of incident cases and prevalent cases. Microbiol Immunol 2007; 51(9): 823-832

10. Fang H, Chen J, Hu J. Modelling the SARS epidemic by a latticebased Monte-Carlo simulation. Conf Proc IEEE Eng Med Biol Soc 2005; 2005: 7470-7473

11. Read JM, Bridgen JR, Cummings DA, Ho A, Jewell CP. Novel coronavirus 2019-nCoV: early estimation of epidemiological parameters and epidemic predictions. medRxiv 2020; doi:10.1101/ 2020.01.23.20018549

12. Ai L. Modelling the epidemic trend of the 2019-nCOV outbreak in Hubei Province, China. medRxiv 2020; doi: 10.1101/2020.01.30. 20019828

13. Chang R, Wang H, Zhang S, Wang Z, Dong Y, Tsamlag L, Yu X, Xu C, Yu Y, Long R, Liu N, Chu Q, Wang Y, Xu G, Shen T, Wang S, Deng X, Huang J, Zhang X, Wang H, Cai Y. Phase- and epidemic region-adjusted estimation of the number of coronavirus disease 2019 cases in China. Front Med 2020; 14(2): 199-209

14. Wang H, Wang Z, Dong Y, Chang R, Xu C, Yu X, Zhang S, Tsamlag L, Shang M, Huang J, Wang Y, Xu G, Shen T, Zhang X, Cai Y. Phase-adjusted estimation of the number of coronavirus disease 2019 cases in Wuhan, China. Cell Discov 2020; 6(1): 10

15. National Health Commission of the People's Republic of China. Outbreak notification. 2020. http://www.nhc.gov.cn/xcs/yqtb/ list_gzbd.shtml (in Chinese) (assessed March 27, 2020)

16. Imai N, et al. Report 3: Transmissibility of 2019-nCoV. March 11, 2020. https://www.imperial.ac.uk/mrc-global-infectious-diseaseanalysis/covid-19/report-3-transmissibility-of-covid-19/ (assessed 
March 27, 2020)

17. Li Q, Guan X, Wu P, Wang X, Zhou L, Tong Y, Ren R, Leung KSM, Lau EHY, Wong JY, Xing X, Xiang N, Wu Y, Li C, Chen Q, Li D, Liu T, Zhao J, Liu M, Tu W, Chen C, Jin L, Yang R, Wang Q, Zhou S, Wang R, Liu H, Luo Y, Liu Y, Shao G, Li H, Tao Z, Yang Y, Deng Z, Liu B, Ma Z, Zhang Y, Shi G, Lam TTY, Wu JT, Gao GF, Cowling BJ, Yang B, Leung GM, Feng Z. Early transmission dynamics in Wuhan, China, of novel coronavirus-infected pneumonia. N Engl J Med 2020; 382(13): 1199-1207

18. Tang B, Wang X, Li Q, Bragazzi NL, Tang S, Xiao Y, Wu J. Estimation of the transmission risk of the $2019-\mathrm{nCoV}$ and its implication for public health interventions. J Clin Med 2020; 9(2): E462

19. Ni H. Will the new epidemic "global pandemic" be out of control if countries do not adopt the Chinese model? 2020. https://view.inews. qq.com/w2/20200312A0SFM900?tbkt $=\mathrm{C} 1 \&$ strategy $=$ \&openid $=$ o04IBAMLFztkSyue6FJfsKpVBoIM\&uid $=$ \&refer $=$ wx_hot\&ft $=$ 0 ( in Chinese ) (accessed March 12, 2020)

20. WHO. Coronavirus disease (COVID-2019) situation reports. 2020. https://www.who.int/emergencies/diseases/novel-coronavirus2019/situation-reports (accessed March 27, 2020)

21. Obadia T, Haneef R, Boëlle PY. The R0 package: a toolbox to estimate reproduction numbers for epidemic outbreaks. BMC Med Inform Decis Mak 2012; 12(1): 147

22. Nikbakht R, Baneshi MR, Bahrampour A. Estimation of the basic reproduction number and vaccination coverage of influenza in the United States (2017-18). J Res Health Sci 2018; 18(4): e00427

23. White LF, Wallinga J, Finelli L, Reed C, Riley S, Lipsitch M, Pagano M. Estimation of the reproductive number and the serial interval in early phase of the 2009 influenza $\mathrm{A} / \mathrm{H} 1 \mathrm{~N} 1$ pandemic in the USA. Influenza Other Respir Viruses 2009; 3(6): 267-276

24. Cori A, Ferguson NM, Fraser C, Cauchemez S. A new framework and software to estimate time-varying reproduction numbers during epidemics. Am J Epidemiol 2013; 178(9): 1505-1512

25. WHO Ebola Response Team, Aylward B, Barboza P, Bawo L, Bertherat E, Bilivogui P, Blake I, Brennan R, Briand S, Chakauya JM, Chitala K, Conteh RM, Cori A, Croisier A, Dangou JM, Diallo B, Donnelly CA, Dye C, Eckmanns T, Ferguson NM, Formenty P, Fuhrer C, Fukuda K, Garske T, Gasasira A, Gbanyan S, Graaff P, Heleze E, Jambai A, Jombart T, Kasolo F, Kadiobo AM, Keita S, Kertesz D, Koné M, Lane C, Markoff J, Massaquoi M, Mills H, Mulba JM, Musa E, Myhre J, Nasidi A, Nilles E, Nouvellet P, Nshimirimana D, Nuttall I, Nyenswah T, Olu O, Pendergast S, Perea W, Polonsky J, Riley S, Ronveaux O, Sakoba K, Santhana Gopala Krishnan R, Senga M, Shuaib F, Van Kerkhove MD, Vaz R, Wijekoon Kannangarage N, Yoti Z. Ebola virus disease in West Africa - the first 9 months of the epidemic and forward projections. N Engl J Med 2014; 371(16): 1481-1495

26. Kandel N, Chungong S, Omaar A, Xing J. Health security capacities in the context of COVID-19 outbreak: an analysis of International Health Regulations annual report data from 182 countries. Lancet 2020; 395(10229): 1047-1053

27. Nishiura H, Linton NM, Akhmetzhanov AR. Serial interval of novel coronavirus (COVID-19) infections. Int J Infect Dis 2020; 93: 284 286

28. World Bank. Population, total. 2019. https://data.worldbank.org.cn/
indicator/SP.POP.TOTL?view $=$ chart $($ in Chinese $)($ accessed March 27, 2020)

29. Desai AN, Kraemer MUG, Bhatia S, Cori A, Nouvellet P, Herringer M, Cohn EL, Carrion M, Brownstein JS, Madoff LC, Lassmann B. Real-time epidemic forecasting: challenges and opportunities. Health Secur 2019; 17(4): 268-275

30. Polonsky JA, Baidjoe A, Kamvar ZN, Cori A, Durski K, Edmunds WJ, Eggo RM, Funk S, Kaiser L, Keating P, de Waroux OLP, Marks M, Moraga P, Morgan O, Nouvellet P, Ratnayake R, Roberts CH, Whitworth J, Jombart T. Outbreak analytics: a developing data science for informing the response to emerging pathogens. Philos Trans R Soc Lond B Biol Sci 2019; 374(1776): 20180276

31. The Lancet. COVID-19: learning from experience. Lancet 2020; 395(10229): 1011

32. Zhang W, Du RH, Li B, Zheng XS, Yang XL, Hu B, Wang YY, Xiao GF, Yan B, Shi ZL, Zhou P. Molecular and serological investigation of 2019-nCoV infected patients: implication of multiple shedding routes. Emerg Microbes Infect 2020; 9(1): 386389

33. The Lancet. COVID-19: too little, too late? Lancet 2020; 395 (10226): 755

34. Li Z, Wu M, Yao JW, Guo J, Liao X, Song SJ, Li JL, Duan GJ, Zhou YX, Wu XJ, Zhou ZS, Wang TJ, Hu M, Chen XX, Fu Y, Lei C, Dong HL, Xu CO, Hu YH, Han M, Zhou Y, Jia HB, Chen XW, Yan JA. Caution on kidney dysfunctions of COVID-19 patients. medRxiv 2020; doi: 10.1101/2020.02.08.20021212

35. Zhou G, Chen S, Chen Z. Back to the spring of 2020: facts and hope of COVID-19 outbreak. Front Med 2020; 14(2): 113-116

36. Li H, Zhou Y, Zhang M, Wang H, Zhao Q, Liu J. Updated approaches against SARS-CoV-2. Antimicrob Agents Chemother 2020. [Epub ahead of print] doi: 10.1128/AAC.00483-20

37. Li G, De Clercq E. Therapeutic options for the 2019 novel coronavirus (2019-nCoV). Nat Rev Drug Discov 2020; 19(3): 149150

38. Chen C, Zhang XR, Ju ZY, He WF. Advances in the research of cytokine storm mechanism induced by coronavirus disease 2019 and the corresponding immunotherapies. Chin J Burns (Zhonghua Shao Shang Za Zhi) 2020; 36(0): E005 (in Chinese)

39. Sina News. The Indonesian President Joko Widodo has appealed to religious leaders to help fight the disease, refusing to close the city but ordering widespread testing. 2020. http://mil.news.sina.com.cn/ 2020-03-20/doc-iimxyqwa1937282.shtml (in Chinese) (assessed March 20, 2020)

40. Wang JB. India has introduced 15 measures to prevent the spread of COVID-19. 2020. http://m.news.cctv.com/2020/03/17/ARTIm4nX L2N8idlYyb5N8NrS200317.shtml (in Chinese) (assessed March 17, 2020)

41. WHO. Do your part to stop stigma and combat COVID-19. 2020. https://www.who.int/emergencies/diseases/novel-coronavirus2019/events-as-they-happen (assessed February 25, 2020).

42. Getz WM, Salter R, Mgbara W. Adequacy of SEIR models when epidemics have spatial structure: Ebola in Sierra Leone. Philos Trans R Soc Lond B Biol Sci 2019; 374(1775): 20180282

43. Bukhari Q, Jameel Y. Will coronavirus pandemic diminish by summer? SSRN 2020; doi: 10.2139/ssrn.3556998 (accessed March 27, 2020) 